\title{
Study on the Characteristics of LFM Signals, BC Signals and Their Mixed Modulation Signals
}

\author{
Zheng Dou, Weidong Song, Wenxu Zhang \\ College of Information and Communication Engineering, Harbin Engineering University, Harbin, China \\ Email: douzheng@hrbeu.edu.cn, songweidong@hrbeu.edu.cn,zhangwenxu@hrbeu.edu.cn
}

How to cite this paper: Dou, Z., Song, W.D. and Zhang, W.X. (2017) Study on the Characteristics of LFM Signals, BC Signals and Their Mixed Modulation Signals. Int. J. Communications, Network and System Sciences, 10, 196-205.

https://doi.org/10.4236/ijcns.2017.108B021

Received: May 30, 2017

Accepted: August 11, 2017

Published: August 14, 2017

\begin{abstract}
This paper proposes a linear frequency modulation (LFM signal) and biphase coding (BC signal) mixed modulation signal called LFM-BC signal. LFM-BC signal has both LFM signal and BC signal two kinds of traditional signal advantages but makes up for their shortcomings. In this paper, LFM-BC signal, LFM and BC signals are studied and compared from the time characteristic and frequency characteristic of the signal, fuzzy function, pulse compression and Doppler characteristics and low probability of interception (LPI) characteristics.
\end{abstract}

\section{Keywords}

LFM Signal, BC Signal, LFM-BC Signal, Pulse Compression, LPI, Doppler Characteristics

\section{Introduction}

LFM signal and BC signal are often used in pulsed radar. LFM signal has the lower side of the sidelobe after pulse compression, and its Doppler frequency is not sensitive, but the signal form is relatively simple, vulnerable to interference. As the $\mathrm{BC}$ signal can use code agile technology, $\mathrm{BC}$ signal anti-jamming performance is better, but the $\mathrm{BC}$ signal is more sensitive to Doppler frequency [1].

In order to integrate the advantages of LFM and BC signals, make up for the shortcomings of the two, this paper proposes a mixed modulation signal with intra-linear chirp and inter-pulse phase coding. This hybrid modulation signal has both LFM and BC signal advantages, but also makes up for their own shortcomings. LFM-BC signal obtains large signal to noise ratio to improve by small time and narrow bandwidth, to avoid the large time and wide bandwidth problems [2].

In this paper, we study and compare the mixed-modulation signal and the 
LFM and BC signal from the aspects of the time characteristic and frequency characteristic, fuzzy functions, pulse compression and Doppler characteristics, low intercepting characteristics, in order to verify the application of mixedmodulation signals.

\section{Signal Time Domain and Frequency Domain Characteristics}

\subsection{Signal Time Domain Characteristic}

The time-domain expression of the LFM signal is as follows:

$$
u_{L F M}(t)=\frac{1}{\sqrt{T}} \operatorname{rect}\left(\frac{t}{T}\right) \exp \left(j 2 \pi k t^{2}\right) \exp \left(\mathrm{j} 2 \pi f_{0} \mathrm{t}\right)
$$

where $T$ is the pulse width, $k=B / T$ is called the frequency modulation slope, $B$ is the signal bandwidth.

The time-domain expression of the $\mathrm{BC}$ signal is as follows:

$$
u_{B C}(t)=\left\{\begin{array}{cl}
\frac{1}{\sqrt{P}} \sum_{k=0}^{P-1} q_{k} v(t-k T), & 0<t<P T \\
0, & \text { others }
\end{array}\right.
$$

where $T$ is the sub-pulse width, $q_{k}$ is the value of the $k$ th code ( 1 or -1$), P$ is the symbol length, and $v(t)$ is the symbol waveform.

At the same time $u_{B C}(t)$ can also be written

$$
u_{B C}(\mathrm{t})=\mathrm{v}(\mathrm{t}) \otimes \frac{1}{\sqrt{P}} \sum_{k=0}^{P-1} q_{k} \delta(t-k T)=u_{1}(t) \otimes u_{2}(t)
$$

where

$$
\begin{aligned}
& u_{1}(t)=v(t)= \begin{cases}1, & 0<t<T \\
0, & \text { others }\end{cases} \\
& u_{2}(t)=\frac{1}{\sqrt{P}} \sum_{k=0}^{P-1} q_{k} \delta(t-k T)
\end{aligned}
$$

mixed modulation signal with intra-linear chirp and inter-pulse phase coding can be expressed as:

$$
u(t)=u_{L F M}(t) \otimes u_{B C}(t)
$$

\subsection{Signal Frequency Domain Characteristic}

The signal frequency domain expression can be obtained from the time domain expression of the signal and the Fourier transform property.

The frequency domain expression of the LFM signal is:

$$
\mathrm{U}_{L F M}(f)=\frac{1}{\sqrt{T}} \exp \left(-j \pi f^{2} / k\right) \int_{-T / 2}^{T / 2} \exp \left(-j\left(\frac{\pi}{2}\right) 2 k\left(t-\frac{f}{k}\right)^{2}\right) d t
$$

The frequency domain expression of the $\mathrm{BC}$ signal:

$$
U_{B C}(f)=U_{1}(f) U_{2}(f)=\sqrt{\frac{T}{P}} \operatorname{sinc}(f T) e^{j \pi f T} \sum_{k=0}^{P-1} q_{k} e^{-j 2 \pi f k T}
$$


where

$$
\begin{gathered}
U_{1}(f)=\sqrt{T} \operatorname{sinc}(f T) e^{-j \pi f T} \\
U_{2}(f)=\sqrt{\frac{1}{P}} \sum_{k=0}^{P-1} q_{k} e^{-j 2 \pi f k T}
\end{gathered}
$$

Since the mixed modulation signal corresponds to the convolution of the LFM signal and the BC signal in the time domain, the frequency domain is the product of the two spectra [3]. So the mixed-modulation signal (LFM-BC) frequency domain expression is

$$
\begin{gathered}
U(f)=U_{L F M}(f) U_{B C}(f) \\
U(f)=U_{L F M}(f) \sqrt{\frac{1}{P} \sum_{k=0}^{P-1} q_{k} e^{-j 2 \pi f k T}}
\end{gathered}
$$

It can be seen from the formula that the spectrum of the mixed modulation signal depends mainly on the shape of $U_{L F M}(f)$, that is, the spectrum of the mixed modulation signal is similar to the spectrum of $U_{L F M}(f)$, As for the additional factor $\frac{1}{\sqrt{P}} \sum_{k=0}^{P-1} q_{k} e^{-j 2 \pi f k T}$, it is related to the form of the code used, it is only in the original LFM signal on the spectrum to bring some glitches, not too much to change its spectral shape.

\section{Fuzzy Function Graph}

The $s(t)$ fuzzy function is defined as the square $\left|\chi\left(\tau ; f_{d}\right)\right|^{2}$ of the two-dimensional cross-correlation function. The exact expression is

$$
\left|\chi\left(\tau ; f_{d}\right)\right|^{2}=\left|\int_{-\infty}^{\infty} s(t) s^{*}(t-\tau) \exp \left(j 2 \pi f_{d} t\right) d t\right|^{2}
$$

According to the definition of fuzzy function, the fuzzy function of LFM signal is:

$$
\left|\chi\left(\tau ; f_{d}\right)\right|^{2}=\left|\left(1-\frac{|\tau|}{\tau^{\prime}}\right) \frac{\sin \left(\pi \tau^{\prime}\left(\mu \tau+f_{d}\right)\left(1-\frac{|\tau|}{\tau^{\prime}}\right)\right.}{\pi \tau^{\prime}\left(\mu \tau+f_{d}\right)\left(1-\frac{|\tau|}{\tau^{\prime}}\right)}\right|^{2} \quad|\tau| \leq \tau^{\prime}
$$

The fuzzy function of the $\mathrm{BC}$ signal is expressed as:

$$
\chi(\tau ; \xi)=\chi_{1}(\tau ; \xi) \otimes \chi_{2}(\tau ; \xi)=\sum_{m=-(P-1)}^{P-1} \chi_{1}(\tau-m T ; \xi) \chi_{2}(m T ; \xi)
$$

where, $\chi_{1}(\tau ; \xi)$ is a fuzzy function of $u_{1}(t)$, and $\chi_{2}(\tau ; \xi)$ is a fuzzy function of $u_{2}(t)$.

$$
\chi_{1}(y ; \xi)= \begin{cases}\exp [j \pi \xi(T-|y|)] \frac{\sin [\pi \xi(T-|y|)]}{\pi \xi(T-|y|)}(T-|y|), & |\tau| \leq T \\ 0, & |\tau| \leq T\end{cases}
$$




$$
\chi_{2}(m T ; \xi)= \begin{cases}\frac{1}{P} \sum_{k=0}^{P-1-m} q_{k} q_{k+m} \exp (j 2 \pi k \xi T), & 0 \leq m \leq(P-1) \\ \frac{1}{P} \sum_{k=-m}^{P-1} q_{k} q_{k+m} \exp (j 2 \pi k \xi T), & -(P-1) \leq m \leq 0\end{cases}
$$

Substituting $y=\tau-m T$ into the formula, we can get the fuzzy function of the $\mathrm{BC}$ signal.

The fuzzy function $\chi(\tau ; \xi)$ of the mixed modulation signal is the convolution of the LFM signal fuzzy function $\chi_{L F M}(\tau ; \xi)$ and LFM signal fuzzy function $\chi_{B C}(\tau ; \xi)$ according to the fuzzy function multiplication rule.

$$
\chi(\tau ; \xi)=\chi_{L F M}(\tau ; \xi) \otimes \chi_{B C}(\tau ; \xi)
$$

The following figure shows the fuzzy functions of the three signals.

It can be seen from Figure 1 that the fuzzy function graph of the LFM signal is tilted blade type, and has the origin symmetry and obtains the maximum value at the origin.

Figure 2 is the fuzzy function graph of the 13-bit Barker code; we can see that the fuzzy function graph center is similar to the pin-type [4].

Figure 3 is the fuzzy function graph of the LFM-BC, we can see that the fuzzy

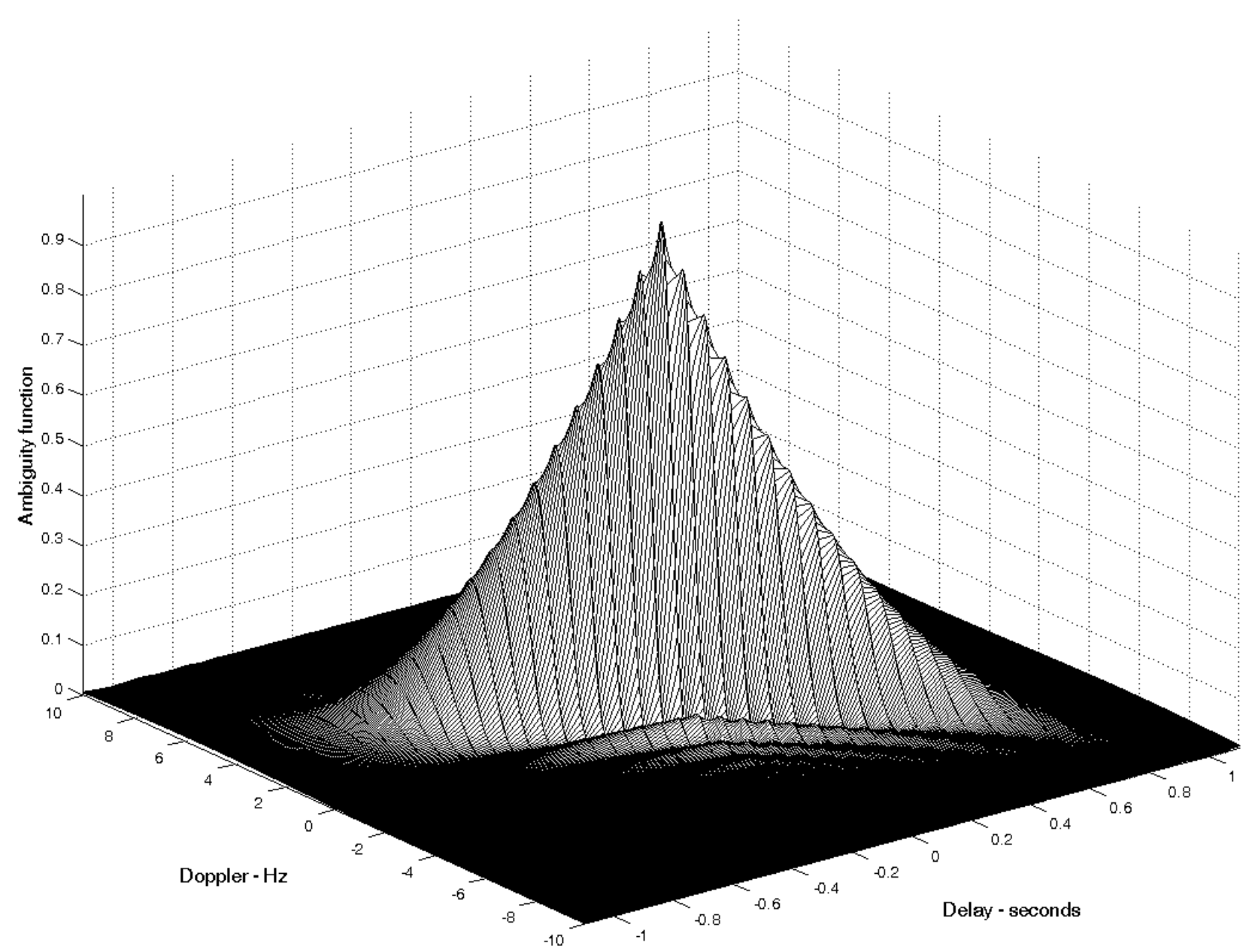

Figure 1. The fuzzy function graph of the LFM signal. 


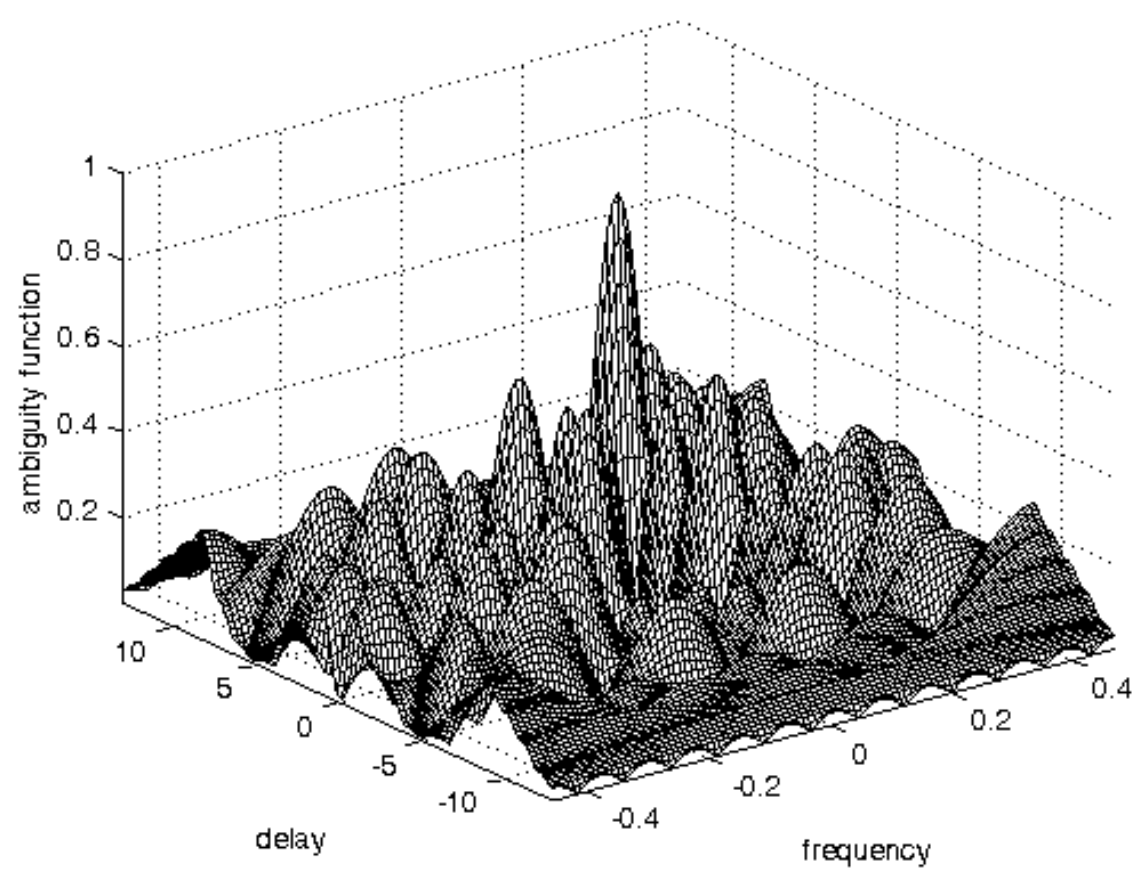

Figure 2. The fuzzy function graph of the 13-bit Barker code signal.

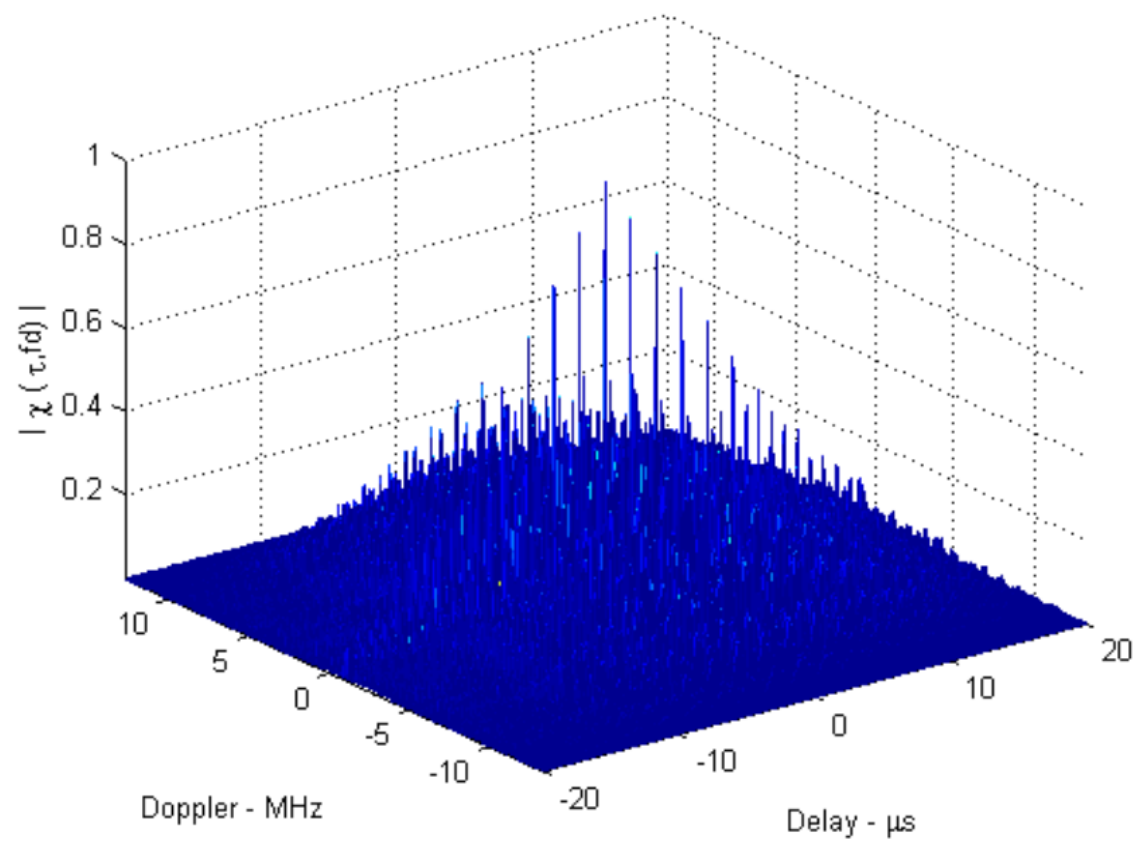

Figure 3. The fuzzy function graph of the LFM-BC signal.

function graph center is similar to the pin-type. The LFM-BC has good measurement properties.

\section{Pulse Compression Results and Doppler Characteristics}

\subsection{LFM Signal Pulse Compression Results and Doppler} Characteristics

When the LFM signal has the Doppler frequency of $f_{d}$, the output of the 
matched filter is:

$$
y(t)=\sqrt{k T^{2}} \frac{\sin 2 \pi\left(f_{d}+k t\right) T / 2}{2 \pi\left(f_{d}+k t\right) T / 2} \exp \left[j 2 \pi\left(-k t^{2} / 2\right)\right] \exp (j \pi / 4)
$$

The above equation shows that when $f \_d=0$, the output pulse has a sinc function type envelope. When $\mathrm{f} \_\mathrm{d} \neq 0$, the sinc function envelope will produce a displacement, causing the ranging error, while the output pulse amplitude slightly decreased [5].

Generally radar LFM pulse waveform has a coupling between the Doppler frequency and the measured distance. That is, when the measured target exists Doppler frequency, the radar measurement target distance and the target where the real distance there is a certain difference.

The following figure illustrates the effect of Doppler frequency on pulse output. The following figure shows the pulse output when the Doppler frequency $f_{d}$ is $0 \mathrm{~Hz}, 60 \mathrm{kHz}, 100 \mathrm{kHz}, 200 \mathrm{kHz}$.

It can be seen from Figure 4 that the main sidelobe ratio of the LFM signal is reduced by Doppler shift, but the effect is not obvious. That is, when there is a large Doppler frequency, it also can get a large compression ratio. That is, LFM signal is not sensitive to Doppler frequency.

\subsection{BC Signal Pulse Compression Results and Doppler Characteristics}

The output of the BC signal through the matched filter is its autocorrelation function $\Phi(m)$,

$$
\Phi(m)=\sum_{k=1}^{N} q_{k} q_{k+m}
$$

In this paper, 13-bit Barker code as an example to analyze the two-phase pulse pressure output. Barker code is a binary pseudo-random sequence code $\left\{q_{n}\right\}$,
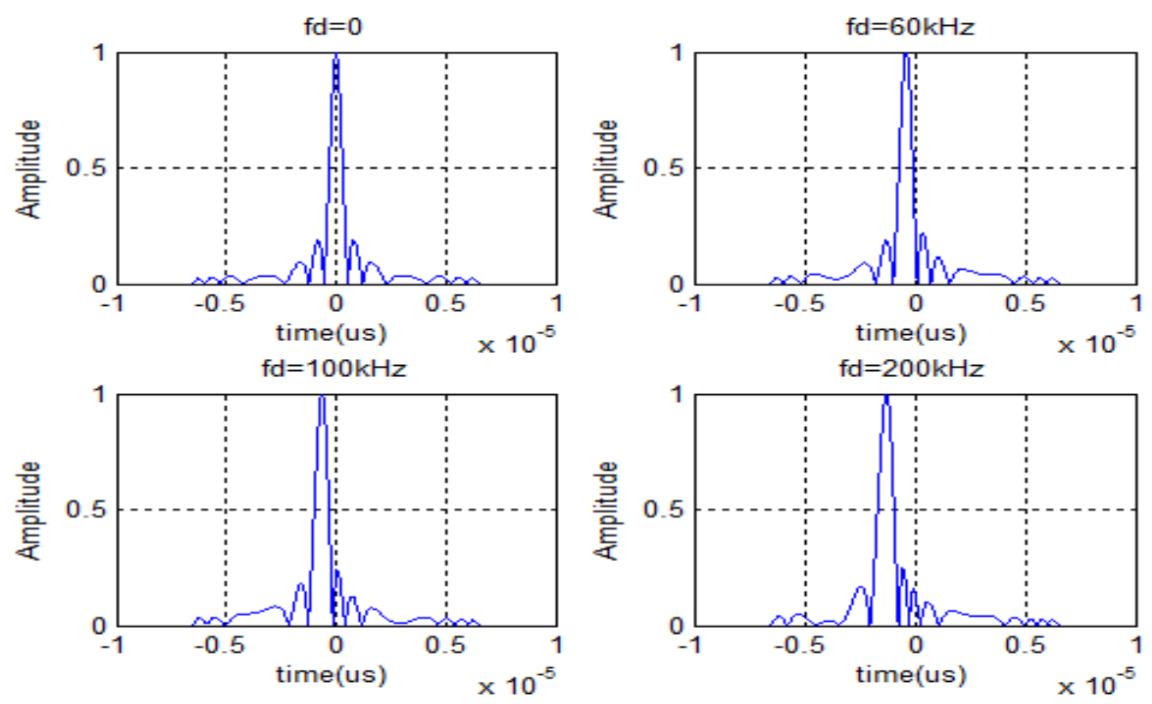

Figure 4. Simulation of LFM signal pulse compression under different doppler frequency shift. 
$q_{n} \in(+1,-1), \mathrm{n}=0,1, \cdots, \mathrm{P}-1$. Its nonperiodic autocorrelation function is satisfied.

$$
\mathrm{R}(\mathrm{m}, 0)=\sum_{k=0}^{P-1-|m|} q_{k} q_{k+m}= \begin{cases}P, & m=0 \\ 0 \text { or } \pm 1, & m \neq 0\end{cases}
$$

The following figure shows the pulse output when the Doppler frequency $f_{d}$ is $0 \mathrm{~Hz}, 60 \mathrm{kHz}, 100 \mathrm{kHz}, 200 \mathrm{kHz}$.

It can be seen from Figure 5 that the peak of the BC pulse output signal does not shift due to the Doppler frequency, but the peak decreases much as the Doppler frequency increases. So the BC signal is more sensitive to Doppler frequency, only applies when the Doppler frequency is small.

\subsection{LFM-BC Signal Pulse Compression Results and Doppler Characteristics}

The impulse response of the LFM-BC signal is,

$$
\mathrm{h}(t)=u^{*}(T P-t)=\int_{-\infty}^{+\infty} u_{L F M}^{*}(\tau) u_{B C}^{*}(T P-t-\tau) d \tau
$$

Let $\tau=T-x$,

$$
\begin{gathered}
h(t)=\int_{-\infty}^{+\infty} u_{L F M}^{*}(T-x) u_{B C}^{*}(T P-t-T+x) d x \\
h(t)=u_{L F M}^{*}(T-t) \otimes u_{B C}^{*}[(P-1) T-t]
\end{gathered}
$$

It can be seen from the formula, the LFM-BC signal matching filter is exactly the LFM signal matched filter and the BC signal matched filter impulse response convolution.

The frequency domain is expressed as,

$$
\mathrm{H}(f)=H_{L F M}(f) \cdot H_{B C}(f)
$$

We can divide the compression process into two steps: The first step through
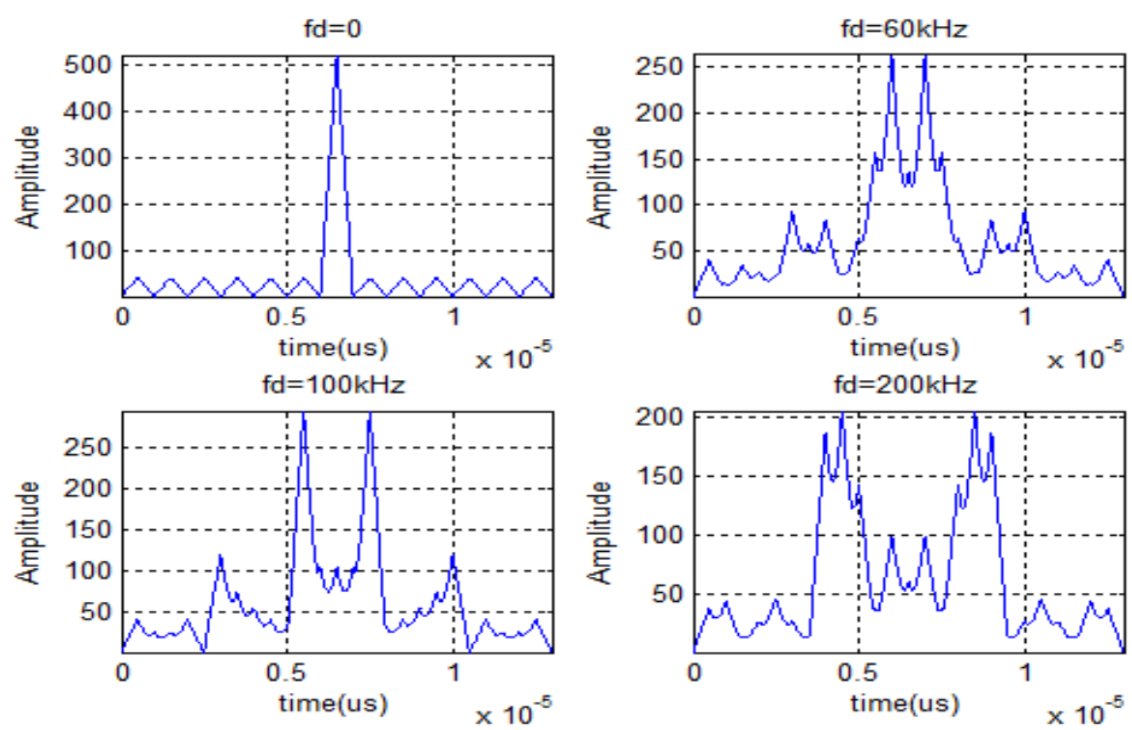

Figure 5. Simulation of BC signal pulse compression under different doppler frequency shift. 
the LFM filter, get the $\mathrm{BC}$ signal. The second step is then through the $\mathrm{BC}$ matched filter to obtain the final pulse compression signal. The two-step pulse compression process can also be done in one step.

The following figure shows the pulse output when the Doppler frequency $f_{d}$ is $0 \mathrm{~Hz}, 60 \mathrm{kHz}, 100 \mathrm{kHz}, 200 \mathrm{kHz}$.

It can be seen from Figure 6 that the LFM-BC signal is basically insensitive to the Doppler signal, As a new type of pulse compression signal, it has the advantages of two signals, but also to make up for their own deficiencies.

\section{LPI Characteristics Analysis}

The intercept factor $\alpha$ is a measure of the degree to which the radar signal is intercepted. The smaller the $\alpha$ indicates that the signal is more difficult to intercept. When the radar uses ultra-low sidelobe antenna, other parameters are certain, and intercept the receiver parameters are also certain. The intercept factor $\alpha$ is only inversely proportional to the square root of the bandwidth and time wide product of the transmitted waveform. That is $\alpha=\mathrm{K}\left(\frac{1}{T B}\right)^{1 / 2}$, where $T$ is the signal width, $B$ is the adjusted signal bandwidth, $K$ is the combined effect of other factors [6].

Let the chirp width of the chirp signal be $T_{1}$ and the bandwidth is $B_{1}$, then the intercepting factor is: $\alpha_{L F M}=\mathrm{K}\left(\frac{1}{T_{1} B_{1}}\right)^{1 / 2}$.

Set the number of BC signal bit $P$, sub-pulse width is still $T_{1}$. The time width of the $\mathrm{BC}$ signal is $T=P T_{1}$. The spectrum of the $\mathrm{BC}$ signal depends primarily on the spectrum of the sub-pulse. So the BC signal bandwidth is approximately
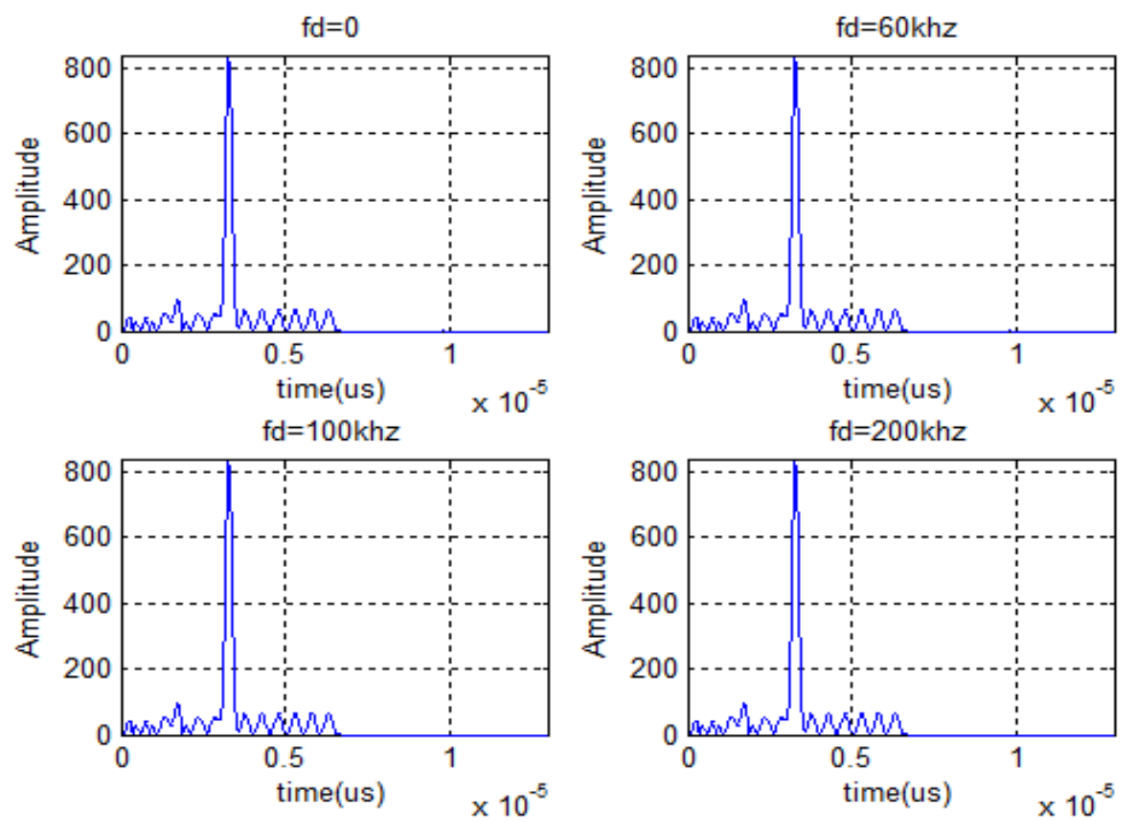

Figure 6. Simulation of LFM-BC signal pulse compression under different doppler frequency shift. 
$B_{2}=1 / T_{1}$, The intercept probability factor is: $\alpha_{B C}=\mathrm{K}\left(\frac{1}{T B_{2}}\right)^{1 / 2}=\mathrm{K}\left(\frac{1}{P}\right)^{1 / 2}$.

LFM-BC signal first pulse within the pulse frequency, and then pulse phase coding. So the time width of the LFM-BC signal is $\mathrm{T}=\mathrm{PT}_{1}$, the bandwidth is similar to the bandwidth $B_{1}$ of the LFM signal. The interception factor of the mixed modulation signal is:

$$
\alpha_{L F M-B C}=\mathrm{K}\left(\frac{1}{T B_{1}}\right)^{1 / 2}=\mathrm{K}\left(\frac{1}{P T_{1} B_{1}}\right)^{1 / 2}=\alpha_{L F M} \frac{1}{\sqrt{P}}=\alpha_{B C} \frac{1}{\sqrt{T_{1} B_{1}}}
$$

That is, the interception factor of the LFM-BC signal is $\frac{1}{\sqrt{P}}$ times of the LFM signal, $\frac{1}{\sqrt{T_{1} B_{1}}}$ times of the BC signal. It can be seen that the longer the $\mathrm{BC}$ code length, the wider the bandwidth of the LFM signal, the more obvious the improvement of the intercept factor of the LFM-BC signal [7].

The LFM-BC signal has the smallest intercept factor, so the signal has better low intercept performance. It can prevent the signal from being intercepted by the receiver to detect and intercept, thereby enhancing the radar in the battlefield combat capability and viability.

\section{Conclusion}

The mixed modulation signal (LFM-BC signal) of LFM signal and BC signal proposed in this paper inherits the advantages of two kinds of single modulation methods, and has some improvement. The implementation of this combination is relatively simple, is a practical combination of signal. At the same time, Doppler shift has little effect on LFM-BC signal. The LFM-BC signal requires a smaller code length than the pure phase code, and the required time-bandwidth product is smaller than the LFM. So the LFM-BC signal obtains a large signal to noise ratio improved by small time and narrow bandwidth. In addition, the LFM-BC signal has a more complex form of signal, making it difficult to extract the radar by the radar intercept receiver. This is the signal characteristic required by the LPI radar.

\section{Acknowledgements}

This work is supported partly by National Natural Science Foundation of China under Grant No. 61301205 and No. 61571146, National Defense Based Science Research Pro-gram under Grant No. JCKY2013604B001. This paper is funded by the International Exchange Program of Harbin Engineering University for Innovation-oriented Talents Cultivation.

\section{References}

[1] Chen, B.X. (2012) Analysis and Design of Modern Radar System. Xi'an University of Electronic Science and Technology Press.

[2] Shi, L., Peng, Y. and Zhang, Y.F. (2003) A Low Intercept Probability Radar Signal 
and Its Signal Processing. 25, 26-28.

[3] Wu, S.J. and Mei, X.C. (2008) Radar Signal Processing and Data Processing Technology. Electronic Industry Press.

[4] Bassem, R., Atef, M. and Elsherbeni, Z. (2016) Radar System Design MATLAB Simulation. Electronic Industry Press.

[5] Zhang, Y.Q., Xu, L. and Li, J. (2006) A Combined Modulated Radar Signal with Low Interception Characteristics. 18, 90-93.

[6] Ding, L.F., Geng, F.L. and Chen, J.C. (2016) Radar Principle. Electronic Industry Press.

[7] Si, W.J., Mi, S.N., Qu, Z.Y. and Yu, F. (2016) Frequency Measurement Method of Modulated Signals Based on Digital Channelization. 28-33.

Submit or recommend next manuscript to SCIRP and we will provide best service for you:

Accepting pre-submission inquiries through Email, Facebook, LinkedIn, Twitter, etc. A wide selection of journals (inclusive of 9 subjects, more than 200 journals)

Providing 24-hour high-quality service

User-friendly online submission system

Fair and swift peer-review system

Efficient typesetting and proofreading procedure

Display of the result of downloads and visits, as well as the number of cited articles

Maximum dissemination of your research work

Submit your manuscript at: http://papersubmission.scirp.org/

Or contact ijcns@scirp.org 